\title{
Spectrum-efficient Stochastic Channel Assignment for Opportunistic Networks
}

\author{
Mohammad J. Abdel-Rahman*, Fujun Lan**, and Marwan Krunz* \\ * Dept. of Electrical and Computer Engineering, University of Arizona, Tucson, AZ, USA \\ ** Dept. of Systems and Industrial Engineering, University of Arizona, Tucson, AZ, USA \\ \{mjabdelrahman, flan, krunz\}@email.arizona.edu
}

\begin{abstract}
The uncertainty in channel quality due to fading and shadowing along with the unpredictability of primary user (PU) activity make channel assignment in opportunistic spectrum access (OSA) networks quite challenging. In this paper, we propose two per-link channel assignment models under channel uncertainty: a static single-stage and an adaptive two-stage. In the static model, channel assignment is performed once, such that the rate demands are met with a probability greater than a certain threshold. This model is appropriate for a distributed network with no centralized spectrum manager. The adaptive model is a two-stage assignment model, where the initial assignment may be corrected once the uncertainties are partially revealed, such that the excess spectrum is returned back to the spectrum manager. This adaptive model is more appropriate when a centralized spectrum manager is available. Our channel assignment algorithms account for adjacent channel interference (ACI) by introducing guard-bands between adjacent channels that are assigned to different links. These algorithms aim at maximizing the spectral efficiency, considering the impact of guard-bands. The static ACI-aware channel assignment problem is formulated as a chance-constrained stochastic subset-sum problem (CSSP), and the adaptive assignment problem is formulated as a two-stage chance-constrained stochastic subset-sum problem with recourse (CSSPR). We develop heuristic algorithms for both models and test their performance. Preliminary results demonstrate that the proposed heuristic algorithms are highly efficient.
\end{abstract}

Index Terms-Channel assignment, opportunistic spectrum access, spectrum efficiency, stochastic optimization, subset-sum problem.

\section{INTRODUCTION}

Motivated by the need for more efficient utilization of the licensed spectrum and facilitated by recent regulatory polices, significant research has been conducted towards developing cognitive radio (CR) technologies for opportunistic spectrum access (OSA) networks. In an OSA system, secondary users (SUs) employ CRs to access the available spectrum in an opportunistic fashion, without interfering with co-located incumbent users, i.e., primary users (PUs).

Channel quality in OSA networks is uncertain. This is partially due to inherent multi-path fading and shadowing, and partially to the unpredictability of PU activity. Recently, the FCC advocated using a database (DB) to facilitate OSA. According to this approach, the SU acquires the set of available channels in its geographical area through a centralized DB. The DB is mostly concerned with SU-to-PU interference, but not PU-to-SU interference [1]. It declares a channel to be available at a given location if the PU signal transmitted over this channel cannot be successfully decoded by a PU receiver at that location. As indicated in [2], the decodability threshold for a digital TV signal is $\sim 15 \mathrm{~dB}$, which means that the TV signal is $\sim 32$ times stronger than the noise level at the limit of the reception range. Therefore, even if a channel is declared available by the $\mathrm{DB}$, this does not mean that it is completely clean, and it can still have a substantial "pollution" due to PU transmissions. Hence, even with the DB approach, PU activity still impacts SU transmissions. The dynamics of this activity causes the channel quality of an OSA network to be uncertain.

In this paper, we propose two (sequential) channel assignment models under channel uncertainty. In the first (static) model, channel assignment is performed in a single stage such that the rate demand of an SU link is satisfied with a probability greater than a certain threshold. This model is appropriate for a distributed network with no centralized spectrum manager. The second model is a two-stage (adaptive) assignment model. In the first stage, channel assignment is performed assuming probabilistically modeled uncertainty in channels quality. After uncertainties in channel rates are partially revealed, when SUs start using the selected channels, they return the excess spectrum (if any) to the spectrum manager. The returned spectrum can then be used by other links in the network. This potentially increases the number of satisfied links in a resource-constrained network. The adaptive model is more efficient when a centralized spectrum manager is available.

In addition to channel uncertainty, our stochastic channel assignment models consider two other aspects. They support channel bonding and aggregation, and they account for adjacent channel interference (ACI). Channel bonding refers to the bundling of multiple adjacent channels, which can then be treated as a single frequency block whose data rate is approximately the sum of the data rates of the individual channels. On the other hand, bundling multiple non-adjacent frequency channels is referred to as channel aggregation. Channel bonding and aggregation have been adopted by the recent IEEE $802.11 \mathrm{n}$ and the upcoming IEEE 802.11 ac standards [3]. ACI is a form of power leakage from adjacent channels due to imperfections in the design of filters and amplifiers in the radio device. The impact of ACI on network throughput was demonstrated in [4].

Most existing channel assignment algorithms do not account for ACI, and assume no power leakage between adjacent chan- 
nels. To mitigate ACI, guard-bands are needed between adjacent channels that belong to different SUs. Introducing guard bands constrains the effective use of the spectrum. In [5], the authors proposed spectrum-efficient guard-band-aware (GBA) channel assignment mechanisms under two scenarios: guardband reuse and no guard-band reuse. In the first model, guardbands can be shared between adjacent transmissions, whereas in the second model, two adjacent transmissions require two distinct guard-bands. The GBA channel assignment mechanisms in [5] do not necessarily result in the optimal spectrum efficiency (i.e., minimum number of newly introduced guard bands). Adopting the guard-band reuse model, an optimal spectrum-efficient GBA channel assignment mechanism was proposed in [6]. The channel assignment mechanism in [6] was formulated as a subset-sum problem (SSP) [7], assuming that the maximum transmission rates supported by various channels are deterministic. In this paper, we consider the more general case where channel rates are random with a known discrete distribution, and we develop two channel assignment schemes for this environment. The discrete rate distribution is obtained from the well-known staircase relationship between the rate and signal-to-interference-plus-noise ratio (SINR), assuming the channel model (i.e., SINR distribution) is known.

Main Contributions-Our main contributions in this paper are summarized as follows:

1. We formulate and solve a probabilistically constrained single-stage ACI-aware channel assignment problem for OSA networks under channel uncertainty. This problem is first formulated as a chance-constrained stochastic subsetsum problem (CSSP). Then, the chance constraint is reformulated, and the CSSP problem is transformed into a binary integer linear program (BILP).

2. We formulate and solve a probabilistically constrained twostage ACI-aware channel assignment problem under channel uncertainty. The problem is first formulated as a twostage chance-constrained stochastic subset-sum problem with recourse (CSSPR). The chance constraint is used to restrict the probability of not satisfying the link demand, whereas the second stage is added to prevent over-satisfying the link demand. The CSSPR is then reformulated as a BILP.

3. We design computationally efficient heuristic algorithms for the CSSP and CSSPR problems.

4. Numerically, we evaluate the heuristic algorithms and compare them with the optimal solutions. Our results show that the proposed heuristics are close-to-optimal.

To the best of our knowledge, this is the first paper that studies the CSSP and CSSPR problems for the discrete distribution case.

Paper Organization-The rest of this paper is organized as follows. In Section II, we present the system model followed by the problem statement. The static and adaptive channel assignment problems are formulated in Section III. The optimal channel assignment models are reformulated in Section IV as BILPs. Heuristic algorithms are also developed in Section IV.

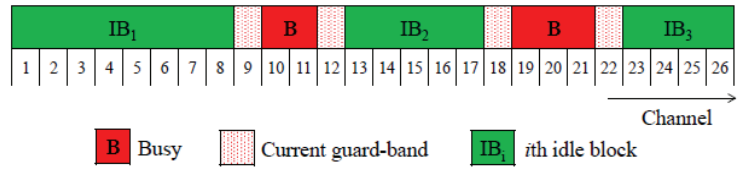

Fig. 1: Example of a spectrum status.

In Section V, we present some numerical results. We conclude the paper in Section VI.

\section{Problem Statement}

Consider an OSA network that operates over $L$ licensed channels. PU activity over each channel is modeled as a continuous-time Markov chain with idle and busy states. For a channel $m$, the transition rates from idle to busy and vice versa are $\lambda^{(m)}$ and $\mu^{(m)}$, respectively. The steady-state probabilities that the PU is idle and busy over channel $m$ are $\pi_{\text {idle }}^{(m)}=\mu^{(m)} /\left(\mu^{(m)}+\lambda^{(m)}\right)$ and $\pi_{\text {busy }}^{(m)}=\lambda^{(m)} /\left(\mu^{(m)}+\lambda^{(m)}\right)$, respectively. The probability that a currently idle channel remains idle during the next $T$ seconds is $p_{i}^{(m)}=e^{-\lambda^{(m)} T}$, and the probability that it becomes busy is $p_{b}^{(m)}=1-e^{-\lambda^{(m)} T}$.

An idle channel can be used as a guard-band (if it is adjacent to a busy channel), or as a data channel otherwise. Assume that the current set of idle channels are grouped into $N$ frequency blocks (after reserving the needed guard-bands). Each block consists of contiguous idle frequency channels. Let $\tilde{R}_{i}, i \in$ $\{1,2, \ldots, N\}$ be a random variable representing the data rate supported by the $i$ th idle frequency block, denoted by $\mathrm{IB}_{i}$. We consider a single-link with a rate demand of $d$ Mbps. Given the current spectrum status, i.e., the state of each of the $L$ licensed channels, our objective is to satisfy the rate demand of the link while maximizing the spectrum efficiency. Figure 1 shows an example of a spectrum status.

The spectrum efficiency $(S E)$ is defined as follows [6]. Let $h_{i}, i \in\{1,2, \ldots, L\}$, be a binary variable indicating whether or not the $i$ th channel is used as a data channel. Similarly, let $\eta_{i}$ be a binary variable indicating whether or not the $i$ th channel is reserved as a guard-band. Then, $S E$ is given by:

$$
S E=\frac{\sum_{i=1}^{L} h_{i}}{\sum_{i=1}^{L} h_{i}+\sum_{i=1}^{L} \eta_{i}} .
$$

It was shown in [6] that in order to minimize the number of newly introduced guard bands, and hence maximize the spectrum efficiency, channels need to be assigned on a perblock basis, instead of the standard per-channel assignment. Considering $N$ idle frequency blocks, $\mathrm{IB}_{1}, \mathrm{IB}_{2}, \ldots, \mathrm{IB}_{N}$, with supported rates $\tilde{R}_{1}, \tilde{R}_{2}, \ldots, \tilde{R}_{N}$, respectively (drawn from a known finite discrete distribution), the question we try to answer is: what is the optimal block assignment strategy for satisfying the link demand $d$ ?

We study the above problem considering two different system models. In the first model, channel block-assignment is performed once and cannot be corrected after the rates are revealed. This corresponds to a network setup where channel assignment is performed for each link independent of other 
links. The second model allows for adjusting the block assignment after the randomness are partially revealed, when nodes start communicating over the assigned blocks. According to the second model, the additional assigned blocks (if any) can be returned back to a centralized spectrum manager, which can then be exploited to satisfy the demands of other links. This dynamic assignment approach increases the number of admitted links in a resource-constrained network, operating under the uncertainty of the quality of its channels.

\section{Problem Formulation}

Let $I=\{1,2, \ldots, N\}$ be the set of indices of the idle frequency blocks and let $x_{i}, i \in I$, be a binary variable; $x_{i}=1$ if the $i$ th frequency block is assigned, and 0 otherwise. Since the rates of the idle frequency blocks (i.e., $\tilde{R}_{i}, i \in I$ ) are random, assigning the blocks only based on their expected rates may result in link under-satisfaction when the actual rates are lower than their expected values, or link over-satisfaction when the actual rates are higher than their expected values. To handle the uncertainty associated with these rates, we propose two different models based on stochastic programming techniques. The first model introduces a chance constraint to restrict the percentage of the occurrence of under-satisfaction, whereas the second model goes a step further, allowing for some corrective action in case of over-satisfaction.

\section{A. Static Single-stage Assignment}

In this section, we formulate the block assignment problem for the first model explained in Section II, where block assignment is performed once (i.e., in a single stage) and correcting the assignment after observing the actual block rates is not allowed. The uncertainty of the rates supported by the frequency blocks causes the feasible region of the assignment problem to be random. Different stochastic optimization approaches have been proposed in the literature to deal with uncertainty in the feasible region of an optimization problem [8]. In this paper, we adopt a chance constraint approach.

As mentioned before, the optimal frequency-block assignment problem was formulated in [6] as a subset-sum problem, assuming deterministic channel rates. In this section, we formulate the assignment problem under channel-rate uncertainty as a chance-constrained stochastic subset-sum problem (CSSP) with a finite discrete distribution. The CSSP formulation is given by:

CSSP:

$$
\begin{gathered}
\underset{x_{i}, i \in I}{\operatorname{minimize}} \sum_{i=1}^{N} \mu_{i} x_{i} \\
\text { subject to } \operatorname{Pr}\left\{\sum_{i=1}^{N} \tilde{R}_{i} x_{i} \geq d\right\} \geq \beta \\
x_{i} \in\{0,1\}, \forall i \in I
\end{gathered}
$$

where $\mu_{i} \stackrel{\text { def }}{=} \mathbb{E}\left[\tilde{R}_{i}\right]$ and $\beta \in(0,1]$ is a prescribed probability. The decision variable $x_{i}, i \in I$, equals one if block $i$ is assigned, and zero otherwise. The objective (2) is to minimize the total expected rate of the assigned blocks, and the chance constraint (3) enforces satisfying the link demand with probability $\geq \beta$. While the chance constraint probabilistically accounts for link under-satisfaction, it does not hedge against the problem of link over-satisfaction. We need to avoid oversatisfying the link, because in a resource-constrained network with multiple links operating in parallel, over-satisfying one link may result in under-satisfying other links in the network.

\section{B. Adaptive Two-stage Assignment}

In this section, we formulate the block assignment problem for the second, adaptive model explained in Section II. The frequency blocks are initially assigned based on their expected rates while satisfying the chance constraint. After the actual rates supported by the assigned blocks are observed, additional blocks (if any) are released and returned to the network spectrum manager. The returned blocks can then be used by other links in the network.

The adaptive assignment problem is formulated as a twostage chance-constrained stochastic subset-sum problem with recourse (CSSPR).

\section{CSSPR:}

$$
\begin{gathered}
\underset{x_{i}, i \in I}{\operatorname{minimize}}\left\{\sum_{i=1}^{N} \mu_{i} x_{i}+\mathbb{E}[h(\boldsymbol{x}, \tilde{\boldsymbol{R}})]\right\} \\
\text { subject to } \operatorname{Pr}\left\{\sum_{i=1}^{N} \tilde{R}_{i} x_{i} \geq d\right\} \geq \beta \\
x_{i} \in\{0,1\}, \forall i \in I
\end{gathered}
$$

where $h(\boldsymbol{x}, \tilde{\boldsymbol{R}})$ is the optimal value of the second-stage problem:

$$
\begin{gathered}
\underset{y_{i}, i \in I}{\operatorname{minimize}}\left\{-\sum_{i=1}^{N} \alpha_{i} \tilde{R}_{i} y_{i}\right\} \\
\text { subject to } y_{i} \leq x_{i}, \forall i \in I \\
\sum_{k=1}^{N}\left(x_{k}-y_{k}\right) \tilde{R}_{k} \geq d y_{i}, \forall i \in I \\
y_{i} \in\{0,1\}, \forall i \in I .
\end{gathered}
$$

The second-stage decision variable $y_{i}, i \in I$, equals one if block $i$ is removed, and zero otherwise. The objective (8) is the (negative of) the total rate of the removed blocks. We assume that the rate of each block $\mathrm{IB}_{i}, i \in I$, at the second stage (i.e., when the block is released after it was previously assigned) is strictly smaller than its first-stage rate, i.e., $0 \leq \alpha_{i}<1$. This way, the later the block is used by a link the smaller the rate this block can support. Setting $\alpha_{i}$ to be strictly less than one for all $i \in I$ avoids having an undesirable aggressive assignment (when $\alpha_{i}=1, \forall i \in I$ ), in which a single link reserves all available blocks and then releases the additional ones. This approach is undesirable because all resources will be allocated to one link first, then all the surplus blocks will be allocated to another link, and so on. Constraint (9) enforces that only blocks that have been assigned in the first stage may be removed, and constraint (10) ensures that a block 
can be removed only when the first-stage assignment has led to an over-satisfaction, and that the link demand remains satisfied after the removal. As in CSSP, a chance constraint is introduced in the first stage to restrict the probability of under-satisfaction.

We note that CSSPR has a relatively complete recourse, i.e., for every feasible first-stage decision $x_{i}$ satisfying (6) and (7), there exists a feasible solution to the second-stage problem under each scenario $\omega \in \Omega$ (e.g., $y_{i}=0, \forall i \in I$ is always a feasible solution to the second-stage problem).

Towards this point, we would like to mention relevant literature dealing with the (static) stochastic knapsack problem. Compared to a subset-sum problem, in a general knapsack problem the value of an item (i.e., its coefficient in the objective function) is different from its weight [7]. To name a few, Kosuch and Lisser [9] presented a chance-constrained model, and a two-stage model with simple recourse, for knapsack problems with random weights. In [10], Kosuch and Lisser formulated a chance-constrained two-stage stochastic program with recourse, where items can be added to or removed from the knapsack in the second stage, when the actual weights become known. However, existing stochastic knapsack formulations either assume a continuous standard distribution (mostly normal) or assume that in the second stage all uncertainties are exposed. In our CSSPR formulation, we assume a finite discrete distribution with known probability mass function (pmf). Moreover, we assume that uncertainties are partially revealed in the second stage, as explained before, which leads us to consider only removing assigned blocks to avoid introducing new uncertainty by adding new blocks.

\section{Problem Reformulation and Solution APPROACH}

In this section, we first present the deterministic equivalent programs (DEPs) for the CSSP and CSSPR problems. The DEP is an equivalent reformulation of the original stochastic program that contains only deterministic variables. We then propose a heuristic algorithm for solving each problem.

\section{A. Static Single-stage Assignment}

\section{1) Deterministic Equivalent Program:}

The DEP of CSSP is given below. Note that the chance constraint in (3) is replaced with the two constraints in (13) and (14), following the same reformulation trick used in the quadratic knapsack formulation in [11]. Constraints (13) and (14) basically say that the sum of the probabilities of the scenarios under which the link demand may not be satisfied is $\leq 1-\beta$, which gives the same meaning as the chance constraint (3).

\section{CSSP (DEP):}

$$
\begin{gathered}
\underset{\substack{x_{i}, u^{(\omega)} \\
i \in I, \omega \in \Omega}}{\operatorname{minimize}} \sum_{i=1}^{N} \mu_{i} x_{i} \\
\text { subject to } \sum_{i=1}^{N} R_{i}^{(\omega)} x_{i} \geq d\left(1-u^{(\omega)}\right), \forall \omega \in \Omega
\end{gathered}
$$

$$
\begin{gathered}
\sum_{\omega \in \Omega} p^{(\omega)} u^{(\omega)} \leq 1-\beta \\
x_{i} \in\{0,1\}, \forall i \in I \\
u^{(\omega)} \in\{0,1\}, \forall \omega \in \Omega
\end{gathered}
$$

where $\Omega$ is the set of scenarios (in here, a scenario represents one realization of the rates of various blocks) and $p^{(\omega)}$ is the probability of scenario $\omega \in \Omega$. $u^{(\omega)}, \omega \in \Omega$, is a binary variable, which equals zero if the block assignment needs to satisfy the demand $d$ under scenario $\omega$, and equals one otherwise. We remark that CSSP (DEP) is a binary integer linear program (BILP).

2) Heuristic Algorithm:

We motivate our heuristic algorithm as follows. Based on Markov's inequality [12], the left-hand-side of the chance constraint (3) can be bounded from above as follows:

$$
\begin{aligned}
\operatorname{Pr}\left\{\sum_{i=1}^{N} \tilde{R}_{i} x_{i} \geq d\right\} \leq & \frac{\mathbb{E}\left[\sum_{i=1}^{N} \tilde{R}_{i} x_{i}\right]}{d} \\
= & \frac{\sum_{i=1}^{N} \mu_{i} x_{i}}{d} .
\end{aligned}
$$

Hence, constraint (3) implies:

$$
\sum_{i=1}^{N} \mu_{i} x_{i} \geq d \beta .
$$

However, (18) does not necessarily imply (3). Therefore, we heuristically make (18) more stringent by multiplying the right-hand-side of (18) with a constant $\kappa>1$ so that constraint (18) becomes:

$$
\sum_{i=1}^{N} \mu_{i} x_{i} \geq \kappa d \beta
$$

The value of $\kappa$ is determined empirically such that (19) best approximates the chance constraint (3). After replacing (3) with (19), CSSP becomes a standard subset-sum problem, which can then be solved efficiently [13].

Our heuristic algorithm can be summarized by the following steps: it was said

1. Replace the chance constraint (3) in CSSP with (19), and solve the resulting (deterministic) subset-sum problem.

2. Evaluate (3) with respect to the obtained solution.

- If (3) is satisfied, terminate.

- Otherwise, modify the current solution by selecting more blocks from the unassigned ones, starting with the block that has the smallest expected rate until a feasible solution to (3) is found. Then, terminate.

- Finally, if assigning all the blocks does not result in a feasible solution, the problem is declared infeasible.

Constraint (3) is evaluated for a given block assignment as follows:

1. We descendingly sort the scenarios according to their probabilities $\left(p^{(\omega)}\right.$ for scenario $\left.\omega \in \Omega\right)$. 
2. Starting from the most probable scenario, we do the following:

- Evaluate the link demand constraint $\sum_{i=1}^{N} R_{i}^{(\omega)} x_{i} \geq d$ under scenario $\omega \in \Omega$.

- If scenario $\omega$ satisfies the link demand constraint, increment the probability of satisfying the link demand by $p^{(\omega)}$.

- If the total probability of satisfying the link demand reaches or exceeds $\beta$, then the given block assignment satisfies (3). Otherwise, go to the next most probable scenario.

3. If all the scenarios have been considered and the total probability of satisfying the link demand is less than $\beta$, then the given block assignment does not satisfy (3).

\section{B. Adaptive Two-stage Assignment}

1) Deterministic Equivalent Program:

The DEP of CSSPR is given below. Similar to CSSP, the chance constraint in (6) is replaced with the constraints in (21) and (22).

CSSPR (DEP):

$$
\begin{gathered}
\underset{\substack{x_{i}, y_{i}^{(\omega)}, u^{(\omega)} \\
i \in I, \omega \in \Omega}}{\operatorname{minimize}}\left\{\sum_{i=1}^{N} \mu_{i} x_{i}-\sum_{\omega \in \Omega} p^{(\omega)}\left(\sum_{i=1}^{N} \alpha_{i} R_{i}^{(\omega)} y_{i}^{(\omega)}\right)\right\} \\
\text { subject to } \sum_{i=1}^{N} R_{i}^{(\omega)} x_{i} \geq d\left(1-u^{(\omega)}\right), \forall \omega \in \Omega \\
\sum_{\omega \in \Omega} p^{(\omega)} u^{(\omega)} \leq 1-\beta \\
y_{i}^{(\omega)} \leq x_{i}, \forall i \in I, \forall \omega \in \Omega \\
\sum_{k=1}^{N}\left(x_{k}-y_{k}^{(\omega)}\right) R_{k}^{(\omega)} \geq d y_{i}^{(\omega)}, \forall i \in I, \forall \omega \in \Omega \\
x_{i} \in\{0,1\}, \forall i \in I \\
y_{i}^{(\omega)} \in\{0,1\}, \forall i \in I, \forall \omega \in \Omega \\
u^{(\omega)} \in\{0,1\}, \forall \omega \in \Omega .
\end{gathered}
$$

Again, CSSPR (DEP) is a BILP.

2) Heuristic Algorithm:

1. Follow the same heuristic approach in Section IV-A2 to get a first-stage feasible solution (i.e., a feasible $x_{i}, i \in I$ ). Otherwise, the problem is declared infeasible.

2. Fixing the first-stage solution, solve the second-stage problem for each scenario. The solution to this problem is used to determine the blocks that need to be excluded from the initial assignment.

We note that the first-stage solution produced by the above heuristic needs be feasible, because, as we mentioned earlier, CSSPR has a relatively complete recourse. Moreover, for a fixed first-stage solution and a specific scenario realization, the second-stage problem reduces to a subset-sum problem.
TABLE I: Rate distribution for five frequency blocks.

\begin{tabular}{|cc|cc|cc|cc|cc|}
\hline $\mathrm{IB}_{1}$ & & $\mathrm{IB}_{2}$ & & $\mathrm{IB}_{3}$ & & $\mathrm{IB}_{4}$ & & $\mathrm{IB}_{5}$ & \\
\hline \hline Rate & Prob. & Rate & Prob. & Rate & Prob. & Rate & Prob. & Rate & Prob. \\
\hline$R_{1}$ & 0.1 & $R_{1}$ & 0.05 & $R_{1}$ & 0.0 & $R_{1}$ & 0.0 & $R_{1}$ & 0.0 \\
$R_{2}$ & 0.8 & $R_{2}$ & 0.1 & $R_{2}$ & 0.05 & $R_{2}$ & 0.05 & $R_{2}$ & 0.0 \\
$R_{3}$ & 0.1 & $R_{3}$ & 0.7 & $R_{3}$ & 0.4 & $R_{3}$ & 0.1 & $R_{3}$ & 0.1 \\
$R_{4}$ & 0.0 & $R_{4}$ & 0.1 & $R_{4}$ & 0.5 & $R_{4}$ & 0.8 & $R_{4}$ & 0.4 \\
$R_{5}$ & 0.0 & $R_{5}$ & 0.05 & $R_{5}$ & 0.05 & $R_{5}$ & 0.05 & $R_{5}$ & 0.5 \\
\hline
\end{tabular}

To see this, rearrange constraint (10) as (28) and consider the set of blocks that have been assigned in the first stage only:

$$
\begin{gathered}
\sum_{\substack{k=1 \\
k \neq i}}^{N} \tilde{R}_{k} y_{k}+\left(\tilde{R}_{i}+d\right) y_{i} \leq \sum_{k=1}^{N} \tilde{R}_{k} x_{k} . \\
\text { V. PERFORMANCE EVALUATION }
\end{gathered}
$$

We numerically evaluate the performance of CSSP and CSSPR. We set $N=5$. Each frequency block can take one of the following rates: $R_{1}=0 \mathrm{Mbps}, R_{2}=1 \mathrm{Mbps}$, $R_{3}=2 \mathrm{Mbps}, R_{4}=4 \mathrm{Mbps}$, and $R_{5}=6 \mathrm{Mbps}$, with the probability distribution shown in Table I. $\alpha_{i}$ is set to 0.8 for all $i \in I$. We solve both CSSP and CSSPR for different combinations of $\beta$ and $d$ values. CPLEX is used to solve the deterministic equivalent programs, CSSP (DEP) and CSSPR (DEP), and obtaining their respective optimal solutions. Our proposed heuristic algorithms produce upper bounds for both models. We set $\kappa=1.5$ in (19) for the heuristics as it was found to be efficient for most instances.

Figure 2 depicts the optimal and the heuristically obtained expected link throughput of CSSP (i.e., the value of the objective function (2)) versus $\beta$ for $d=6,10$, and 14 Mbps. The figure shows that for $\beta \geq 0.7$, the optimal expected link throughput generally exceeds the demand, and that the throughput increases with $\beta$. Moreover, Figure 2 shows that the heuristic solutions are relatively close to the optimum, and at some instances both solutions coincide.

Figure 4 depicts the expected link throughput of CSSPR versus $\beta$ for $d=6,10$, and $14 \mathrm{Mbps}$. The optimal expected link throughput again increases with $\beta$, but more slowly compared with the expected link throughput of CSSP. This is due to the recourse action in the second stage of CSSPR, in which we attempt to remove additional blocks. This results in a smaller net link throughput. Once again, the heuristic solution is found to be relatively close to the optimum. Note that the CSSP and CSSPR problems are both infeasible when $d=14$ Mbps and $\beta>0.7$.

Figures 3 and 5 show the expected link throughput of CSSP and CSSPR, respectively vs. $d$ for $\beta=0.7,0.8$, and 0.9. As can be seen, for large values of $\beta$ and $d$ the CSSP and CSSPR problems turn out to be infeasible. Also, these figures show that the proposed heuristic algorithms achieve a relatively close-to-optimal performance.

\section{CONCLUSIONS}

In this paper, we proposed two channel assignment models under channel uncertainty: a single-stage static assignment 


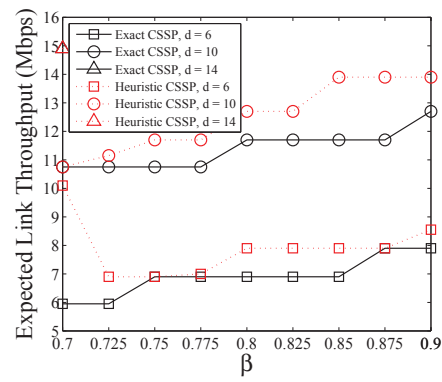

Fig. 2: Expected link throughput vs. $\beta$ for the single-stage channel assignment.

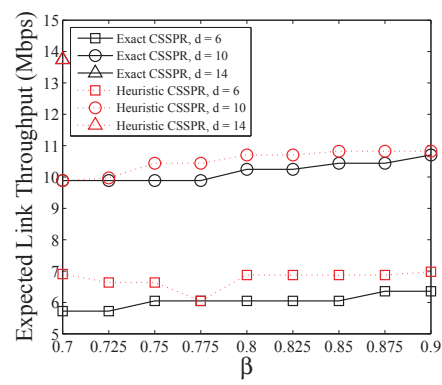

Fig. 4: Expected link throughput vs. $\beta$ for the two-stage channel assignment.

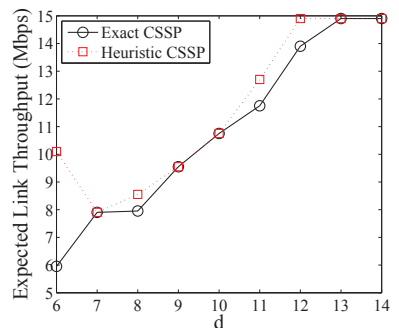

(a) $\beta=0.7$

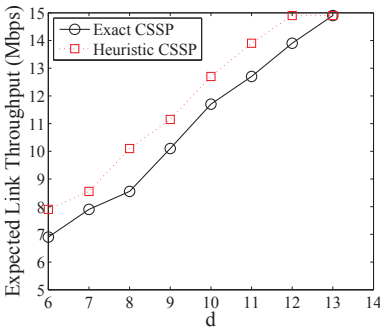

(b) $\beta=0.8$

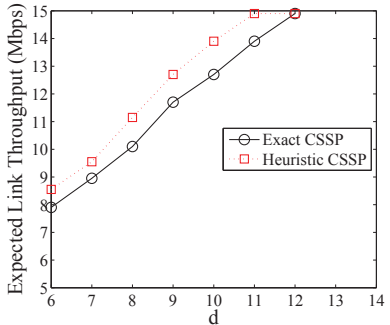

(c) $\beta=0.9$

Fig. 3: Expected link throughput vs. $d$ for the single-stage channel assignment.

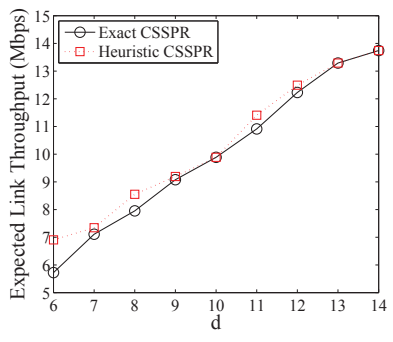

(a) $\beta=0.7$

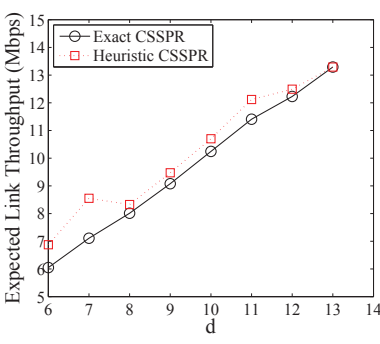

(b) $\beta=0.8$

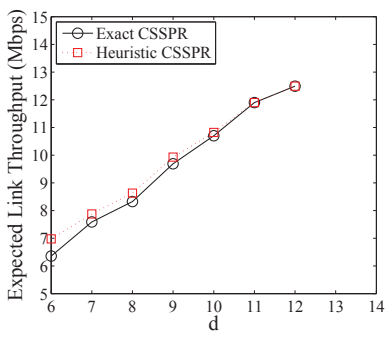

(c) $\beta=0.9$

Fig. 5: Expected link throughput vs. $d$ for the two-stage channel assignment.

model and a two-stage adaptive assignment model. Both assignment models were formulated using stochastic optimization techniques. Optimal solutions for the formulated assignment problems were obtained, and simple but efficient heuristic algorithms were developed. The proposed assignment algorithms also account for adjacent channel interference. The performance of the heuristic algorithms was numerically evaluated against the optimal solution under different values of the model parameters. Our preliminary computational results indicate that the proposed heuristic algorithms are quite efficient.

\section{ACKNOWLEDGMENT}

This research was supported in part by NSF (under grants CNS-1016943 and CNS-0904681, IIP-0832238, IIP-1231043), Raytheon, and the "Connection One" center. Any opinions, findings, conclusions, or recommendations expressed in this paper are those of the author(s) and do not necessarily reflect the views of the National Science Foundation. The authors would like to thank Dr. Güzin Bayraksan, Integrated Systems Engineering Department, The Ohio State University, for her helpful discussions on stochastic optimization.

\section{REFERENCES}

[1] K. Harrison, S. M. Mishra, and A. Sahai, "How much white-space capacity is there?" in Proceedings of the IEEE DySPAN Conference, 2010, pp. 1-10.
[2] Y. Wu, E. Pliszka, B. Caron, P. Bouchard, and G. Chouinard, "Comparison of terrestrial DTV transmission systems: the ATSC 8-VSB, the DVB-T COFDM, and the ISDB-T BST-OFDM," IEEE Transactions on Broadcasting, vol. 46, no. 2, pp. 101-113, 2000.

[3] D. Skordoulis, Q. Ni, H.-H. Chen, A. Stephens, C. Liu, and A. Jamalipour, "IEEE 802.11n MAC frame aggregation mechanisms for nextgeneration high-throughput WLANs," IEEE Transactions on Wireless Communications, vol. 15, no. 1, pp. 40-47, Feb. 2008.

[4] V. Angelakis, S. Papadakis, V. Siris, and A. Traganitis, "Adjacent channel interference in 802.11a is harmful: Testbed validation of a simple quantification model," IEEE Communications Magazine, vol. 49, no. 3, pp. 160-166, March 2011.

[5] H. Bany Salameh, M. Krunz, and D. Manzi, "An efficient guard-bandaware multi-channel spectrum sharing mechanism for dynamic access networks," in Proceedings of the IEEE GLOBECOM Conference, Dec. 2011, pp. $1-5$.

[6] G. S. Uyanik, M. J. Abdel-Rahman, and M. Krunz, "Optimal guardband-aware channel assignment with bonding and aggregation in multichannel systems," to appear in the Proceedings of the IEEE GLOBECOM Conference, Dec. 2013.

[7] S. Martello and P. Toth, Knapsack problems: algorithms and computer implementations. John Wiley \& Sons, 1990.

[8] P. Kall and S. W. Wallace, Stochastic Programming. John Wiley and Sons, 1994.

[9] S. Kosuch and A. Lisser, "Upper bounds for the 0-1 stochastic knapsack problem and a B\&B algorithm," Annals of Operations Research, vol. 176, pp. 77-93, 2010.

[10] S. Kosuch and A. Lisser, "On two-stage stochastic knapsack problems," Discrete Applied Mathematics, vol. 159, pp. 1827-1841, 2011.

[11] A. Lisser and R. Lopez, "Stochastic quadratic knapsack with recourse," Electronic Notes in Discrete Mathematics, vol. 36, pp. 97-104, 2010.

[12] A. Klappenecker, "Markov's inequality." [Online]. Available: http://faculty.cs.tamu.edu/klappi/csce689-s10/markov.pdf.

[13] T. H. Cormen, C. Stein, R. L. Rivest, and C. E. Leiserson, Introduction to Algorithms, 2nd ed. McGraw-Hill Higher Education, 2001. 